\title{
ONLY IMAGE BASED FOR THE 3D METRIC SURVEY OF GOTHIC STRUCTURES BY USING FRAME CAMERAS AND PANORAMIC CAMERAS
}

\author{
A. Pérez Ramos ${ }^{a}$, G. Robleda Prieto ${ }^{a}$ \\ a “G.I.R.A.P.” (“Grupo de Investigación en Representación Arquitectónica del Patrimonio" - Architectonic Representation of \\ Cultural Heritage Research Group) - Department of Architectonic Theory and Representation, Rúa da Fraga 8, Campus da Zapateira, \\ 15008 A Coruña, Spain - araceli.perez.ramos@udc.es
}

Commission V, WG V/2

KEY WORDS: Cultural Heritage, Close Range Photogrammetry, Immersive Photogrammetry, Spherical Photogrammetry, Point Cloud, Virtualization, HDR, 360 Images, Panoramas, Immersive Images, Spherical Images.

\begin{abstract}
:
Indoor Gothic apse provides a complex environment for virtualization using imaging techniques due to its light conditions and architecture. Light entering throw large windows in combination with the apse shape makes difficult to find proper conditions to photo capture for reconstruction purposes. Thus, documentation techniques based on images are usually replaced by scanning techniques inside churches. Nevertheless, the need to use Terrestrial Laser Scanning (TLS) for indoor virtualization means a significant increase in the final surveying cost. So, in most cases, scanning techniques are used to generate dense point clouds. However, many Terrestrial Laser Scanner (TLS) internal cameras are not able to provide colour images or cannot reach the image quality that can be obtained using an external camera. Therefore, external quality images are often used to build high resolution textures of these models. This paper aims to solve the problem posted by virtualizing indoor Gothic churches, making that task more affordable using exclusively techniques base on images. It reviews a previous proposed methodology using a DSRL camera with 18 135 lens commonly used for close range photogrammetry and add another one using a HDR $360^{\circ}$ camera with four lenses that makes the task easier and faster in comparison with the previous one. Fieldwork and office-work are simplified. The proposed methodology provides photographs in such a good conditions for building point clouds and textured meshes. Furthermore, the same imaging resources can be used to generate more deliverables without extra time consuming in the field, for instance, immersive virtual tours. In order to verify the usefulness of the method, it has been decided to apply it to the apse since it is considered one of the most complex elements of Gothic churches and it could be extended to the whole building.
\end{abstract}

\section{INTRODUCTION}

Nowadays, virtualized heritage is used for multiple applications: documentation, monitoring, virtual tours and virtual museums, augmented reality (AR). Aforementioned application usually have in common the need of pre-generate a 3D model. Demanded requirements of these models will vary depending on the application, particularly model resolution (Visitini, 2009).

\subsection{Laser scanning and photogrammetry data acquisition.}

Cultural Heritage data acquisition techniques commonly used are laser scanning and photogrammetry. These techniques are often mixed within a project in order to obtain results required or appropriate to the subsequent application of 3D model. Inside buildings, similar to the case presented, the process commonly used involves using Terrestial Laser Scanners to generate volumes and geometries. However, laser scanner cannot provide colour images or image quality is not enough if we pursue a high resolution textured model. So, external cameras are used to obtain high quality images and build model texture. Methodology that combines laser scanning and photogrammetry provides good results (Visintini, 2009). Nevertheless, laser scanning dependence makes it a high cost technique not available to many professionals.
Furthermore, using both techniques entails doing work twice. Using photogrammetry as unique technique to survey allows volume and texture creation within a single workflow. (Pérez, 2011) Not to mention costs, which are highly reduced.

\subsection{Panoramic images as documentation source.}

The interest in panoramic images as source to heritage documentation has been increased in the last decade. Several approaches have permitted to generate geometries and $3 \mathrm{D}$ models based on panoramic images (d'Annibale, 2009, 2011 and 2013) and Spherical Photogrammetry has been further investigated and improved (Fangi, 2007, 2009, 2013 and 2015). Thus, the metric effectiveness of panoramic images has been validated and the use of these images within virtual environments is already widespread.

However, Image matching technique based on panoramic images is not yet a common practicum and available literature and manuals don't provide guidelines. Furthermore, previous study cases of virtualization using panoramic images pursue image modelling and the majority of those that work with point cloud creation are applied in outdoor environments (Fangi, 2015).

\footnotetext{
* Corresponding author
} 


\subsection{Indoor Ghotic capture features.}

The main problem to virtualize a Gothic building indoors, or similar features architectures, exclusively by image matching techniques lies in difficulty to find proper lighting conditions, which should be as uniform as possible to get adequate restitution images, especially around windows and gaps opened in building walls (Ginovart, 2014) (Pérez Ramos, 2015). This article aims to solve the problem posed by virtualization indoor Gothic churches making that task more affordable exclusively by photogrammetric techniques. The proposed methodology allows obtaining photographs in such a good conditions for virtualizing the target by point cloud. In order to verify the usefulness of the method, It has been decided to apply it to the apse. Since the apse is one of the most complex interior part of these kind of buildings.

\section{SANTIAGO APSE}

The church of Santiago, located in Betanzos (A Coruña, Spain), is in the English way to Santiago de Compostela. The church patron was Fernán Pérez de Andrade "O Mozo". It was reconstructed over a Romanesque previous church having a Ghotic apse which construction was adapted to the previous church structure. Materials used are common in the area: granite quarries close to Betanzos, chestnut wood and tile.

\section{DSRL PROPOSED METHODOLOGY}

A methodology to virtualize indoor Gothic apses by using image based techniques and a DSRL camera has been previously proposed (Pérez Ramos, 2015). A Canon 550 D SRL pre-calibrated by the author and total station Leica TCR370 were used to capture and to rotate, scale and translate the model. Nevertheless, distortion control of the model was no provided.

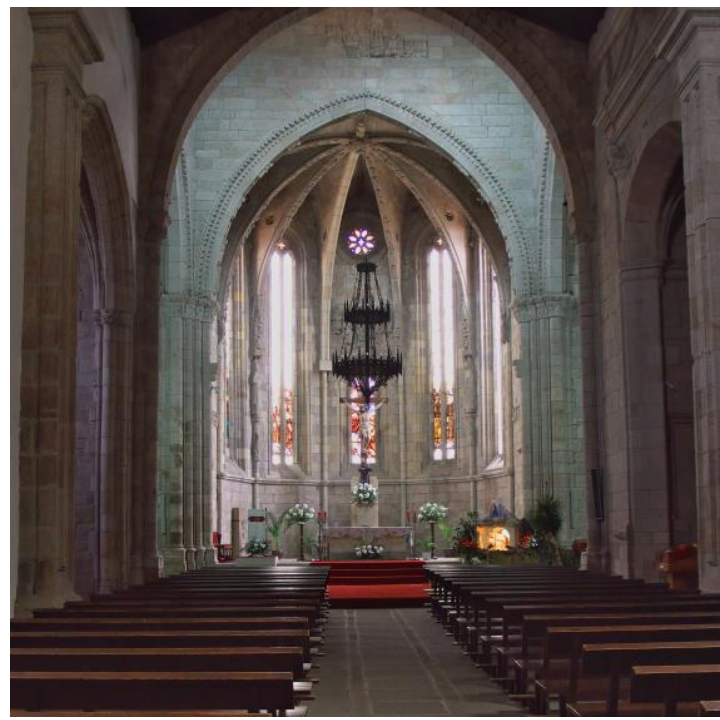

Figure 1. San Francisco Gothic apse. Indoors. (Pérez Ramos, 2015)

Furthermore, auxiliary lightning equipment was needed since single exposure images were used in order to save time at field (capturing) and at office (processing).

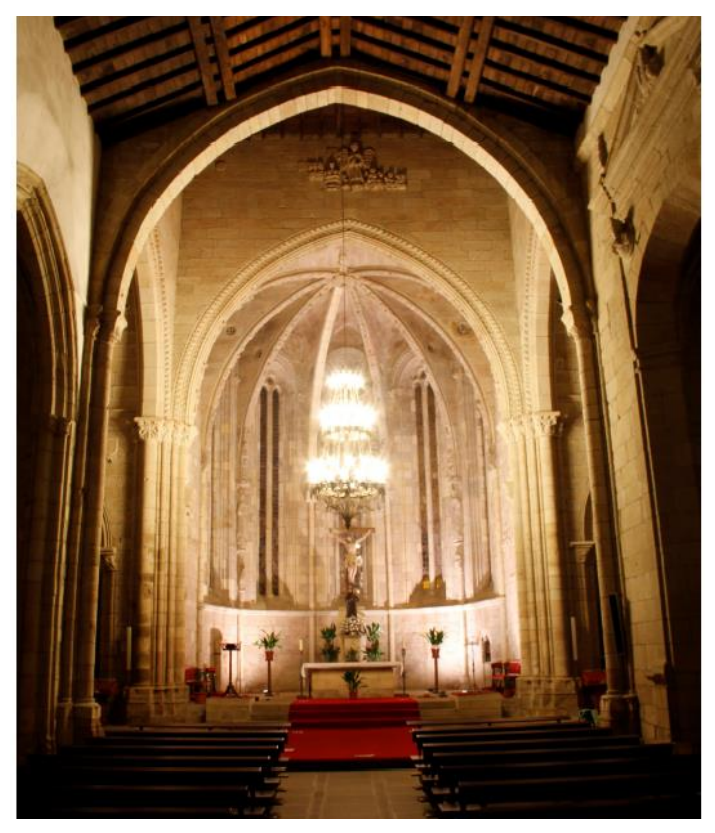

Figure 2. Lighting conditions. San Francisco church settings. (Pérez Ramos, 2015)

The same proposed methodology (Pérez Ramos, 2015) was applied to Santiago's church Gothic apse. Thus, distortion model control is provided in this paper to complement the previous work and to validate the results achieve by using an HDR $360^{\circ}$ camera with 4 lenses mounted.

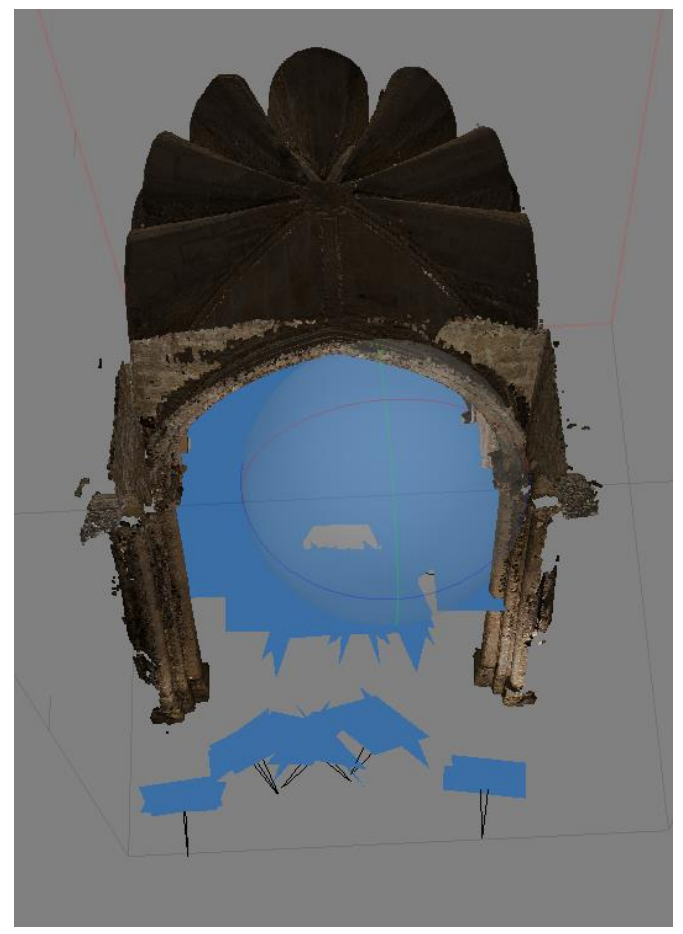

Figure 3. Santiago Ghotic apse. SRL photo capturing. 


\section{HDR 360 METHODOLOGY}

\subsection{Equipment: NCTech iSTAR}

NCTech iSTAR 360 camera is used to capture spherical images $\left(360^{\circ} \times 137.5^{\circ}+/-5^{\circ}\right)$. It's a multi-lens camera with 4 lenses mounted which provides HDR spherical images (50 Mpx). Some of the iSTAR photo-capture features beneficial for indoor image matching techniques are:

4.1.1. High Dynamic Range (HDR): iSTAR HDR images are obtained from exposure fusion method where HDR result is gained pixel by pixel from the source images based on the most appropriate values established by software algorithms. Exposure fusion produces results with less noise and more natural looking appearance, against tone mapping processes which amplify the noise.

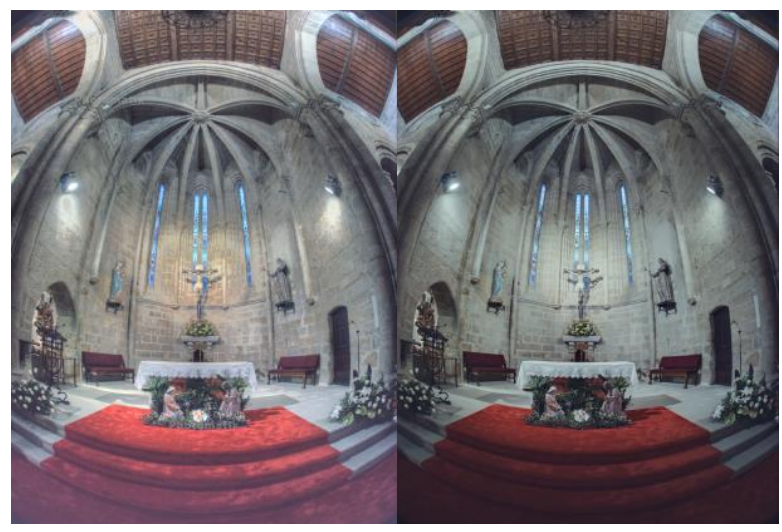

Figure 4. HDR gained from tone mapping in Photomatix (default settings) on the left and HDR gained from exposure fusion in NCTech ImmersiveStudio.

4.1.2. Auto-level function: NCTech Immersive Studio desktop application for iSTAR data processing permits, among other functions, correct iSTAR raw data by automatic auto-levelling function.

4.1.3. Acurate pre-calibration: each iSTAR camera is precision engineered to $0.002 \mathrm{~mm}$ accuracy and calibrated within a manufacturing stage at a sub pixel accuracy by using NCTech algorithm over 800 surveyor targets.

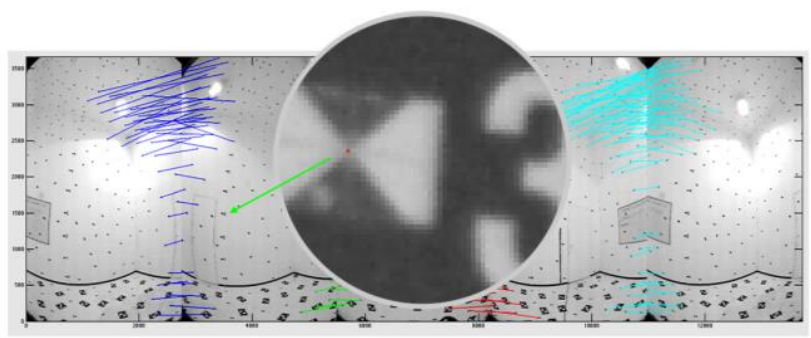

Figure 5. iSTAR calibration (www.nctechimaging.com)

Automatic stitch process: ImmersiveStudio benefits from NCTech calibration processes for automatic and accurate stitching.

\subsection{Photo Capture.}

Planning capture strategy in advanced is a must. You should ensure the number of photos is more than enough avoiding blind areas and image overlapping is $60 \%$ or more.

Auxiliary lightning equipment is no required and the following camera settings were used; ISO 100, HDR ON (5 exposure levels) and "Capture with current analysis" iSTAR mode disabled:

4.2.1. HDR value: $i S T A R$ can be set as HDR OFF (single exposure), HDR ON (5 exposure levels) or HDR PRO (9 exposure levels). General speaking, HDR ON could be more than enough for indoors capturing. However, there are some landscapes, scenes or building rooms with wider range of exposure values. In such cases, HDR PRO will provide better results for building point clouds.

4.2.2."Capture with current analysis" iSTAR mode: This is a default iSTAR setting which activates automatically after taking the first photo. When iSTAR camera takes an image, firstly analyses the whole scene and stablish the proper time exposure value on average to 360 degrees and then it takes the image. Using this setting means analysis time can saved time between captures. However, scenes with very changeable light conditions between shootings may require disable it. Going back one screen by pressing the green arrow on the left up corner is enough to disable it.

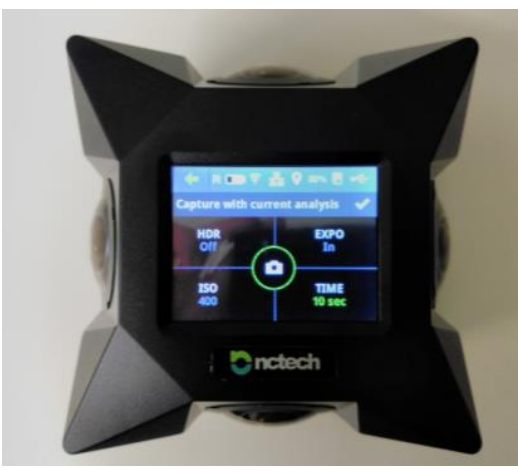

Figure 6. "Capture with current analysis" iSTAR mode activated.

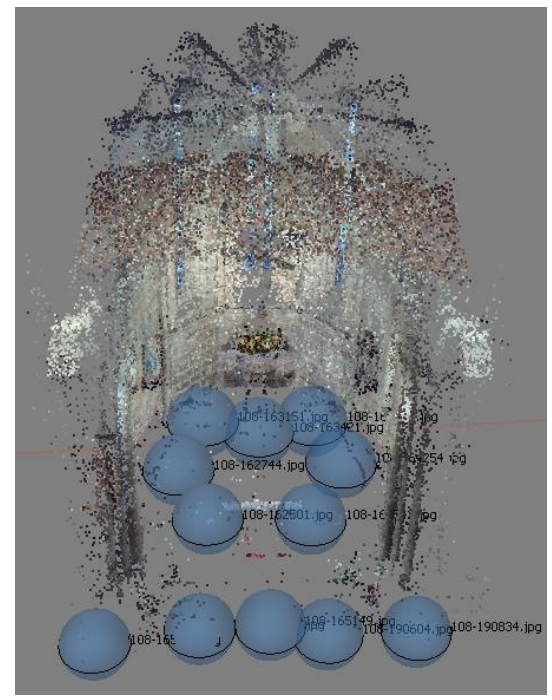

Figure 7. Santiago Ghotic apse. HDR $360^{\circ}$ photo capturing. 


\subsection{Spherical images processing.}

iSTAR raw data is processing within NCTech ImmersiveStudio, which uses NCTech iSTAR calibration file to process stitching. Processing images into NCTech ImmersiveStudio means the user takes advance of this careful calibration in very controlled conditions, since the software uses the calibration parameters provided by NCTech to stitch images. Thus, the error is reduced against stitching without taking advanced of NCTech calibration parameters if $3^{\text {rd }}$ party software is used to stich the images. Furthermore, HDR (among other parameters available) is processed while stitching.

NCTech Immersive Studio can set the output image resolution automatically or it can be selected from several pre-set options $(2 \mathrm{~K}, 4 \mathrm{~K}$ or $16 \mathrm{Mpx})$. In order to get the maximum resolution "Auto" was selected.

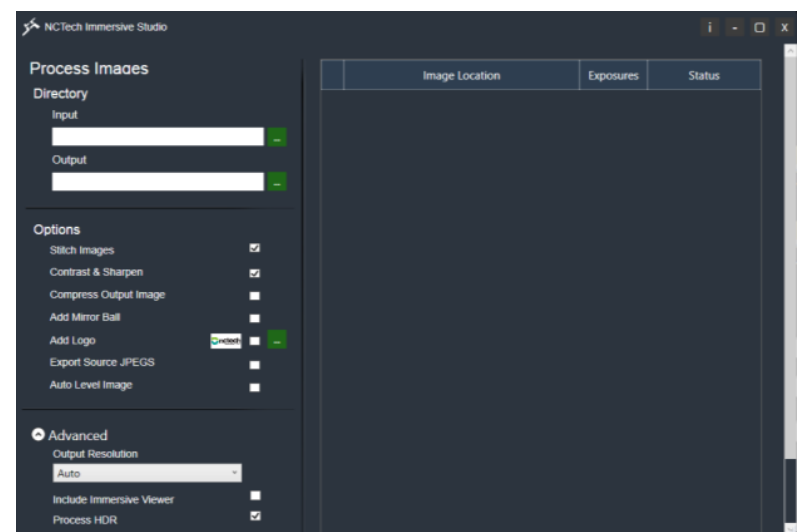

Figure 8. NCTech ImmersiveStudio interface.

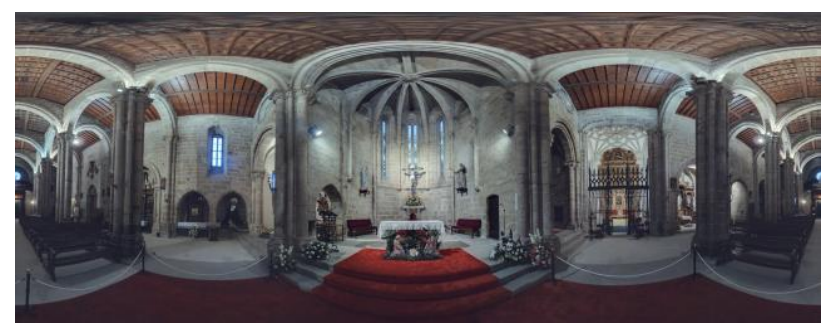

Figure $9.360^{\circ}$ image provided by ImmersiveStudio after processing iSTAR data.

\subsection{Restitution software: Agisoft PhotoScan.}

The Spherical images in equirectangular format gained from NCTech ImmersiveStudio are straightfoward directly imported into Agisoft PhotoScan. Agisoft PhotoScan 1.2.0 build 2198 has been used for point cloud building (medium quality settings), meshing and texturing.

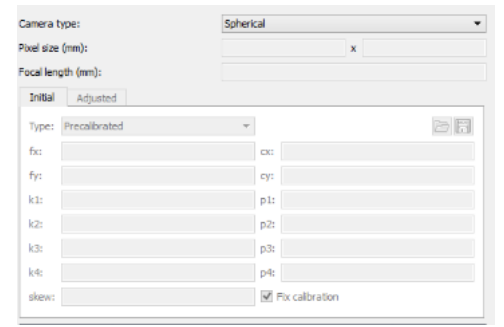

Figure 10. "Camera Calibration" window. Agisoft PhotoScan.
Calibration parameters will appear disabled within Agisoft PhotoScan. Therefore, calibration parameters characteristics of each NCTech iSTAR camera have been applied within NCTech Immersive Studio processing, so after processing it is considering without lens deformation in Agisoft PhotoScan workflow.

\section{LASER SCANNING}

FARO Focus X130 was used to scan the apse (medium point quality settings) in order to get a point cloud to use as reference for accuracy comparison after being registered within FARO SCENE. Four scan stations were needed to scan the apse, three inside the apse and one in front of the main archery.

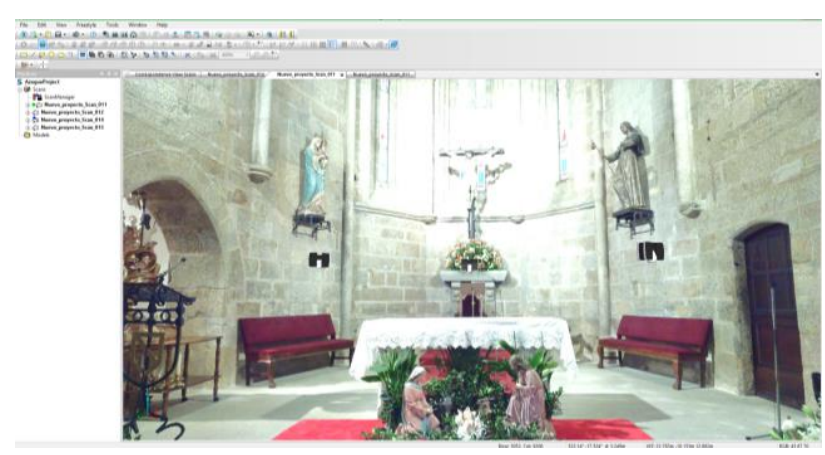

Figure 11. Scan Station 004 "3D View” in FARO SCENE.

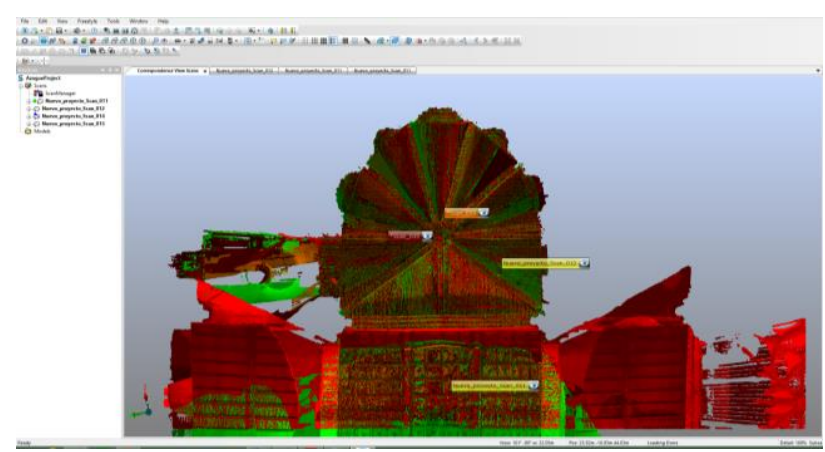

Figure 12. "Correspondence View" of registered scan data in FARO SCENE.

While capturing colour data by using laser scanner internal camera the following message from FARO Focus X130 appears due to site light conditions:

Laser scanner had set in Spanish. Translation from Spanish to English will be:

\section{"1650: Too low environment light"}

"It's possible The scanner was no able to set the proper camera configuration and image quality could be affected. The environment light could be too low or the stablished exposure measurement mode is not the proper one for the currently light conditions..."

Which validate the challenging indoor conditions indoor Ghotic buildings. 


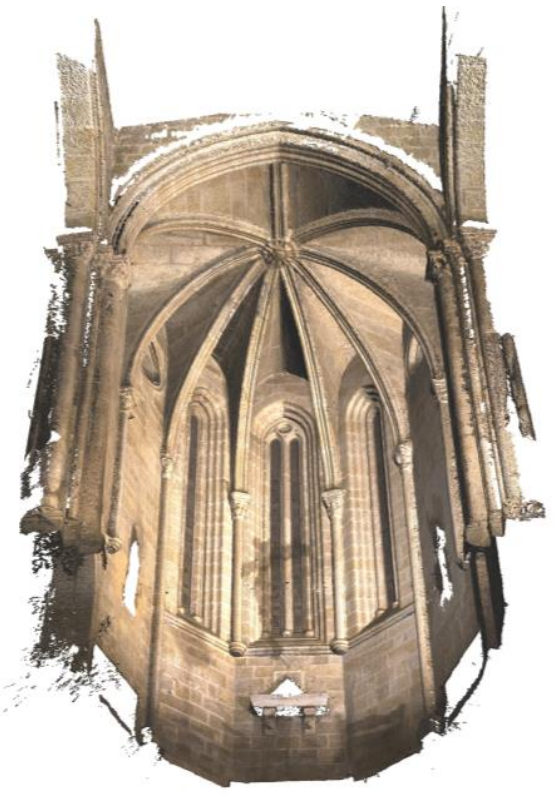

Figure 14. Canon 550 D SRL point cloud.

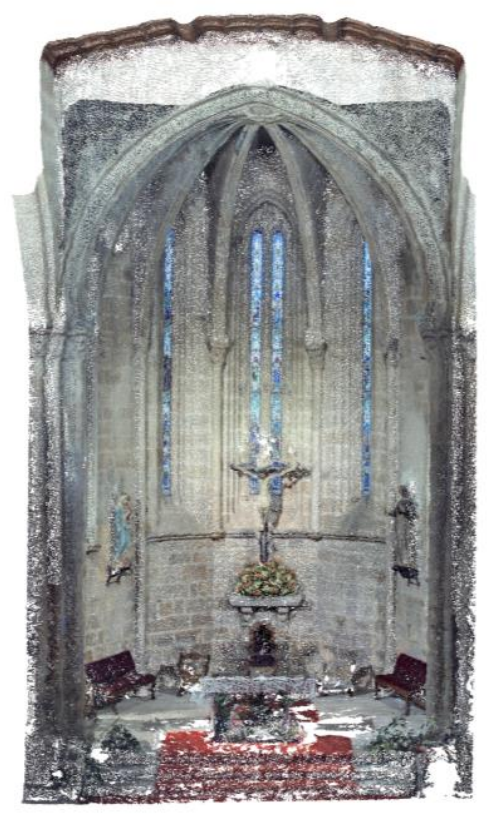

Figure 16. NCTech iSTAR point cloud.

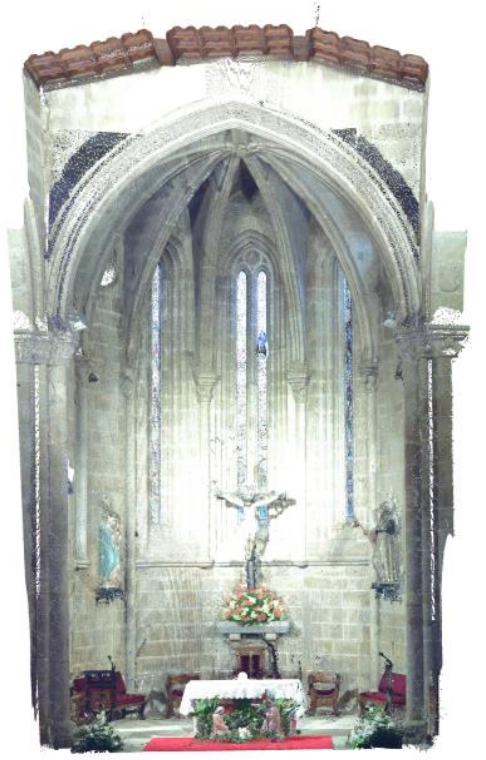

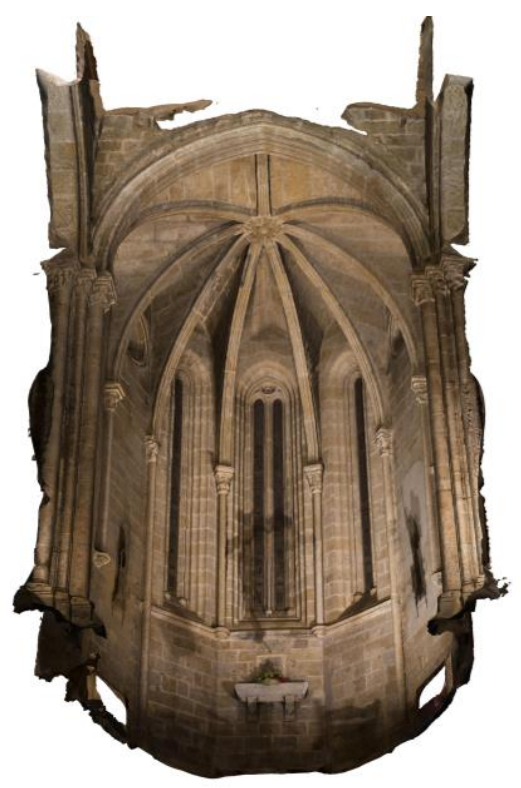

Figure 15. Canon 550 D SRL textured mesh.

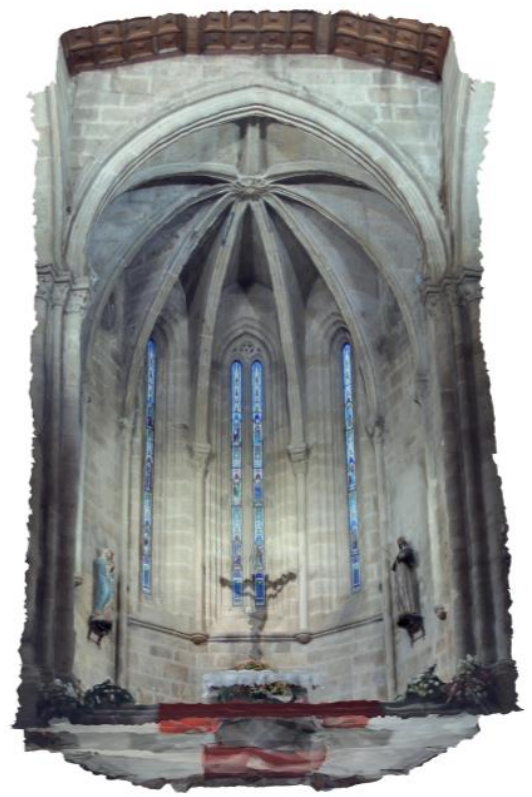

Figure 17. NCTech iSTAR textured mesh.

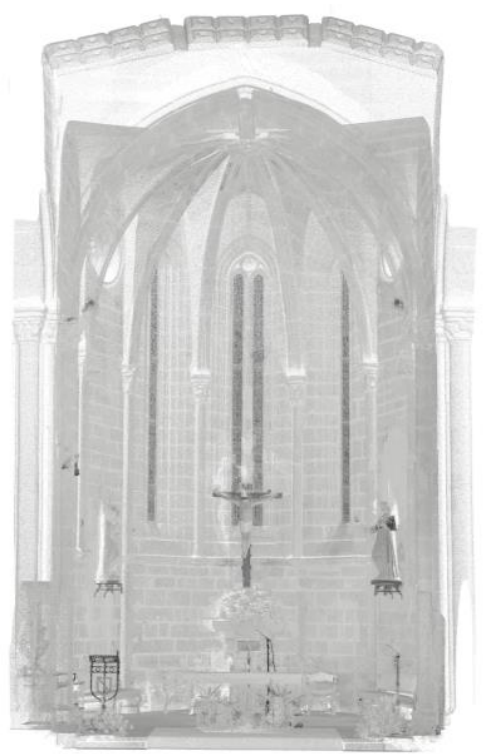

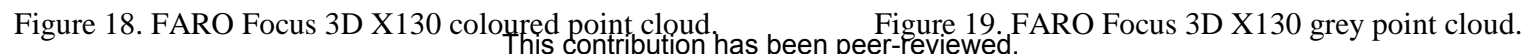




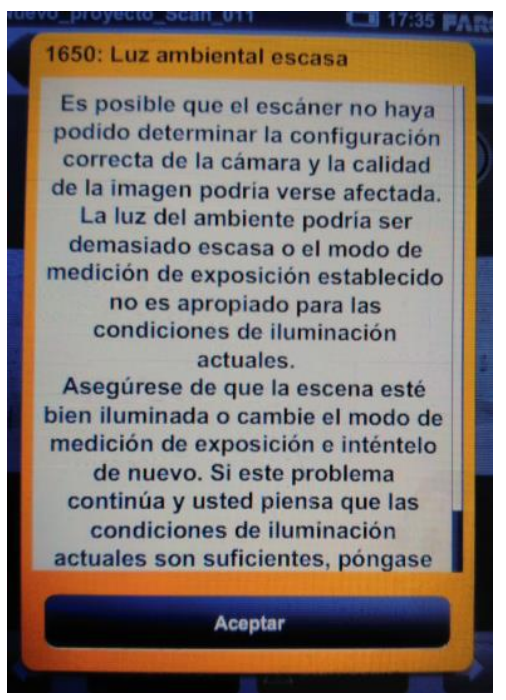

Figure 20. FARO Focus 3D X130 interface. Warning given when colour capturing with laser scanner internal camera in low light conditions

\section{RESULTS}

\subsection{Canon 550 D SRL restitution.}

Coloured point cloud (11.989.171 points, medium quality settings) and textured mesh were obtained by using 60 frame images (18 Mpx). Photo capturing took 2.5 hours.

The data obtained belongs to a previous project. The subsequent analysis required Saints and furniture to be removed, Thus, furniture and Saints are missing is this model. Furthermore, the aim of this paper is the apse restitution and no the elements it contains.

As it is shown in the results, geometric definition is very good, very close to the one obtained by laser scanning. Nevertheless, some strong shadows can be seen on the vaults and near the floor. Furthermore, the colour doesn't reproduce the reality and colourful glass windows aren't represented correctly. Notice that although the scan point number is almost four times the point number provided by Canon 550 D SRL camera within PhotoScan, the resolution is more than enough for general geometry documentation.

\subsection{NCTech iSTAR restitution.}

Coloured point cloud (2.373.124 points, medium quality settings) and textured mesh were obtained by using 12 spherical images (50 Mpx). Photo capturing took $30 \mathrm{~min}$.

The fewer point number can be appreciated if we compare the model with the one provided by Canon 550 D SRL camera but the definition will be enough to document the apse. Notice that the colour provided by iSTAR is nearest to reality and colourful glass window are displayed correctly. Furthermore, there is no strong shadows near the floor and the ones on the vaults are no strong and therefore, details can be appreciated in shadows.

\subsection{FARO Focus 3D X 130 point cloud.}

Coloured point cloud (41.892.875 points after registration and subsampling, medium quality settings) was obtained by using 4 scan stations. Scan data capturing took $50 \mathrm{~min}$.

Laser scan point cloud is the one with higher point number and therefore, better resolution. However, the point cloud had to be decimated and could need to be subsampled to easy performance in $3^{\text {rd }}$ party software (different from laser scanner registration software) and/or powerful computer could be required so that advantage could became a drawback. Furthermore, it shows the worst colour representation, useless in overexposed areas where no detail can be appreciated.

\subsection{Accuracy comparison.}

The point clouds obtained by using image matching techniques are compared to the scan date to validate not only the precision level that can be achieved with these techniques but also de model distortion.

Thus, the range of comparison is set between 0 and $0.05 \mathrm{~m}$ in both cases. At first sight, the point cloud obtained by using Canon 550 D SRL data seems more uniform. The majority of the points showed a distance difference of $0.002 \mathrm{~m}$, being the most common values under $0.025 \mathrm{~m}$.

The results showed by iSTAR-scan data comparison seem less uniform at first sight. Nevertheless, the graphic distribution of values is quite similar. The majority of the points showed distance differences of 0.002 , being most common values under $0.025 \mathrm{~m}$. The graphic is lower because of the lower point cloud resolution.

Following it is show a table for comparison of frame camera methodology against spherical methodology

\begin{tabular}{|r|c|c|}
\hline Camera & Frame & Spherical \\
\hline $\begin{array}{r}\text { Canon 550 D SRL } \\
18-135 \mathrm{~mm} \text { lens }\end{array}$ & NCTech iSTAR \\
\hline $\begin{array}{r}\text { Raw format } \\
\text { processing }\end{array}$ & $\begin{array}{c}\text { Camera RAW } \\
\text { (Manual) }\end{array}$ & $\begin{array}{c}\text { ImmersiveStudio } \\
\text { (Automatic) }\end{array}$ \\
\hline Image resolution & $18 \mathrm{Mpx}$ & $50 \mathrm{Mpx}$ \\
\hline Tripod & yes & yes \\
\hline Auxiliar lightning & yes & no \\
\hline HDR & no & HDR ON \\
& 5 exposures) \\
\hline $\begin{array}{r}\text { Number of images } \\
\text { Time capturing }\end{array}$ & $2.5 \mathrm{hours}$ & $30 \mathrm{~min}$ \\
\hline $\begin{array}{r}\text { PhotoScan } \\
\text { processing }\end{array}$ & $4.85 \mathrm{hours}$ & $45 \mathrm{~min}$ \\
(Medium) & 11.989 .171 & 2.373 .124 \\
\hline $\begin{array}{r}\text { Dense Point Cloud } \\
\text { Model resolution }\end{array}$ & $1.31 \mathrm{~mm} / \mathrm{pix}$ & $4.5 \mathrm{~mm} / \mathrm{pix}$ \\
\hline
\end{tabular}

Table 1. Canon 550 D SRL frames compared to NCTech iSTAR HDR spherical images. 

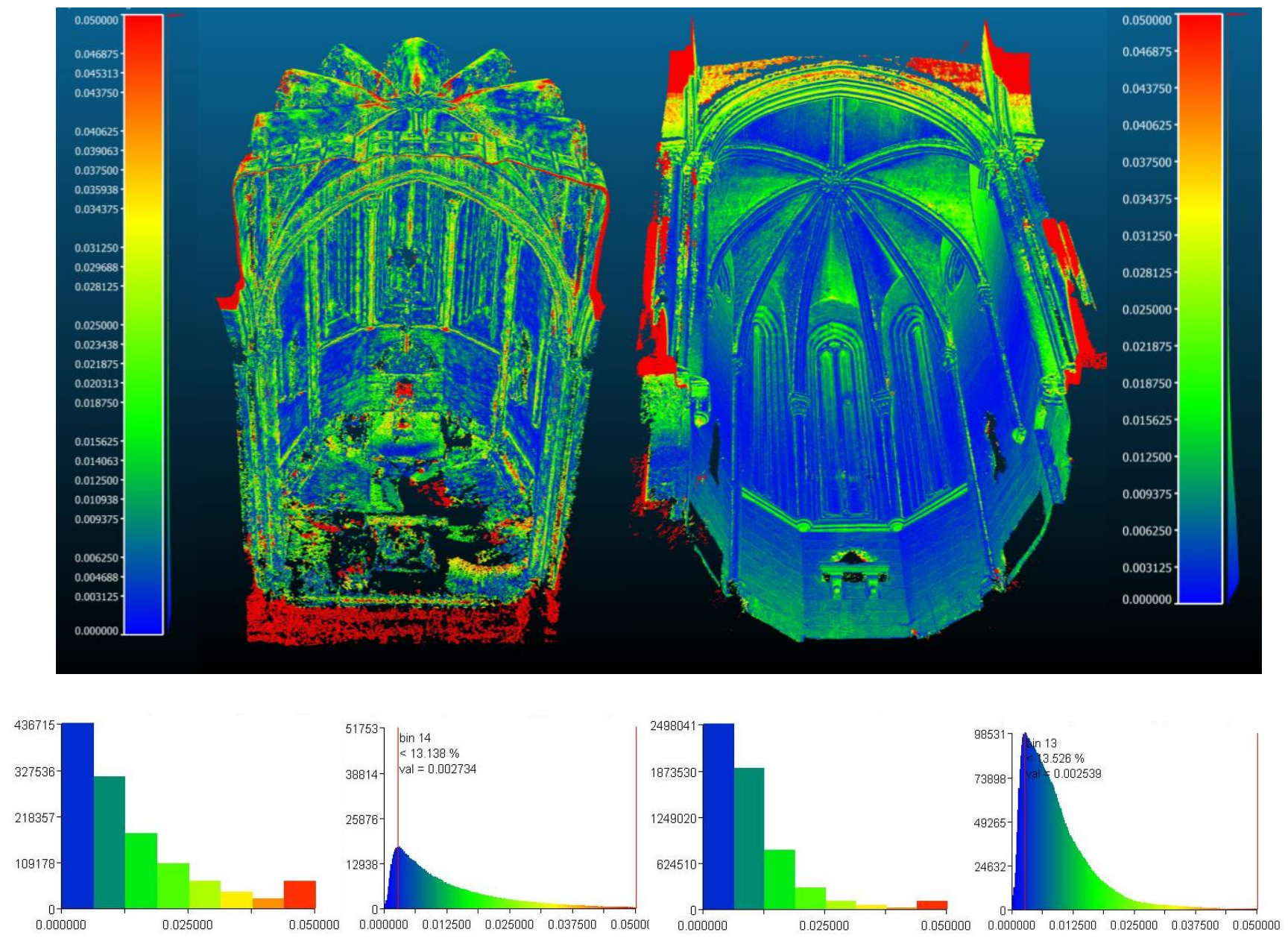

Figure 21. NCTech iSTAR (left) and Canon 550 D SRL (right) point cloud deviation compared to FARO Focus 3D X130.

\section{DISCUSSION}

The interior of Gothic buildings has features that make virtualizing a complex and laborious task. Light entering through windows and other wall holes involves not optimal conditions for photo to restitution. In apses cases, space shape is an added difficulty. Using lighting equipment and careful image development become key points in this sort of work when using single exposure and frame images. Nevertheless using HDR $360^{\circ}$ cameras make the task easier and faster.

Thus, NCTech iSTAR $360^{\circ}$ camera analyses the whole scene and stablish the proper settings considering the scene light conditions which, in combination with the automatic stitching and accurate calibration at manufacture stage, make the task simpler and photo-knowledge becomes less crucial.

iSTAR HDR capabilities make possible the colour representation closer to reality and details available in shadow areas, therefore takes advantage not only against the frame methodology applied (A. Pérez Ramos, 2015) but also against laser scanning. HDR features could be also applied in both, laser scanner colour capture and SRL frame images but capturing and processing times will increase.

Time needed for restitution by using a HDR $360^{\circ}$ camera clearly represent an advantage against both laser scanner (on site) and SRL frame image sources (on site and office).
Point cloud resolution obtained with iSTAR is clearly lower but enough for general geometry documentation. Furthermore, dense point cloud showed is obtained by using "medium" PhotoScan settings and therefore, point cloud with higher density could be obtained if selected "high" or "ultra-high" settings.

Accuracy obtained by using HDR $360^{\circ}$ images shows a similar range of values as using SRL frames, mostly under $0.025 \mathrm{~m}$, being most common value $0.002 \mathrm{~m}$. Note that the accuracy values claim by main laser scanner brands are $+/-2 \mathrm{~mm}$ but registration errors need to be added.

In addition, $360^{\circ}$ images provide the possibility to offer extra deliverables without adding time consuming on site as virtual tours.

\section{CONCLUSION}

Image matching techniques have proved the potential competitive against laser scanning techniques in terms of accuracy since the majority of points have values near the ones claim by main laser scanner brands. Furthermore, when using HDR 360 accurate pre-calibrated cameras, image matching techniques also became competitive in terms of colour quality and time consuming. Although the point cloud resolution is higher when using laser scanning, it could become a drawback if proper computer resources are not available and they usually need to be decimated in order to be able to work with them 
within $3^{\text {rd }}$ party software. Thus, point cloud resolution gained from image matching techniques is enough for both general and detail documentation.

In addition, image matching techniques by using HDR 360 accurate pre-calibrated cameras make the task easier and faster and therefore, image based techniques become competitive when virtualizing indoors Ghotic buildings and architectures with similar features.

\section{ACKNOWLEDGEMENTS}

The authors greatly thanks the whole NCTech Team for lending an iSTAR for data capture and NCTech ImmersiveStudio for data processing, especially Cameron Ure, Neil Tocher, Andrew Baddeley, Chris Dryden, Mansour Ahmadian, Claire Morand, Amirali Matin, Benjie Marwick, Jordan Sewell, Graeme Tinsdale, Dale Borland, Daniele Carotenuto, Rob Jones, Allan Young, Emma Carter, David Munro, Euan Stirling, Deborah Pettit, Dhinesh Ganesan, Craig Duncan, Lee Grant and Thomas Downie for fully support when any doubts arise. Thanks for technical support are given to Agisoft PhotoScan Team, especially Alexey Pasumansky. In addition, we wish to thank GLOBAL Geosystems S.L. especially Carlos Mosquera, Borja Martínez and Angel Montero for his collaboration and equipment lending.

\section{REFERENCES}

d'Annibale, E., Fangi, G., 2009. Interactive modelling by projection of oriented spherical panorama. In : 3D-Arc'2009, $3 D$ Virtual Reconstruction and Visualisation of complex Architectures, ISPRS Archives, Vol. XXXVIII-5/W1.

d'Annibale, E., 2011. Image-Based Modeling from Spherical Photogrammetry and Structure For Motion. The case of the Treasury, Nabatean Architecture in Petra. In : The XXIIIrd International CIPA Symposium, Prague, Czech Republic.

d'Annibale, E., Tassetti, A.N., Malinverni E.S., 2013. From panoramic photos to a low-cost photogrammetric workflow for cultural heritage 3D documentation. In: XXIV International CIPA Symposium, 2 - 6 September 2013, Strasbourg, France, Volume XL-5/W2,

Fangi, G., 2007. The Multi-image spherical Panoramas as a tool for Architectural Survey. In: XXI International CIPA Symposium, ISPRS International Archives, Atene, Greece, Vol XXXVI-5/C53.

Fangi, G., 2009. Further Developments of the Spherical Photogrammetry for Cultural Heritage, 22nd CIPA Symposium. Kyoto, Japan: 11-15 October.

Fangi, G., Nardinocchi, C., 2013, Photogrammetric processing of spherical panoramas. In: The Photogrammetric Record 28(143): 293-311

Fangi, G., 2015, Towards an easier orientation for spherical photogrammetry. In: $3 D$ Virtual Reconstruction and Visualization of Complex Architectures, ISPRS Archives, 25-27 February 2015, Avila, Spain, Volume XL-5/W4, 2015
Ginovart, J.L., Toldrá, J.M., Costa, A., Coll, S., 2014. Close range photogrammetry and constructive characterizacion of masonry Gothic vaults. Journal of Construction 13(1),pp. 47-55

Pérez, A., Cachero, R., Navarro, S., Jordá, F., López, D., Lerma, J. L., Martos, A., 2011. Close range photogrammetry and terrestrial laser scanning: high resolution textured 3D model of the Capel of The Kings in the Palencia Cathedral as a case study. In: XXIIIrd International CIPA Symposium. Prague, Czech Republic. Journal Geoinformatics FCE CTU

Pérez Ramos, A., Robleda Prieto, G., 2015. 3D Virtualization by close range photogrammetry indoor Gothic church apses. The case of study of church of San Francisco in Betanzos (La Coruña, Spain) In: $3 D$ Virtual Reconstruction and Visualization of Complex Architectures, ISPRS Archives, 25-27 February 2015, Avila, Spain, Volume XL-5/W4, 2015

Robleda Prieto, G., Pérez Ramos, A., 2015. Modeling and accuracy assessment for 3D- virtual reconstruction in cultural heritage using low cost photogrammetry: surveying of the "Santa María de Azogue" church's front. In: 3D Virtual Reconstruction and Visualization of Complex Architectures, ISPRS Archives, 25-27 February 2015, Avila, Spain, Volume XL-5/W4, 2015

Visintini, D., Siotto, E., Menean, E., 2009. 3D Modelling of the St. Anthony Abbot Church in S. Daniele del Friuli (I): From laser scanning and photogrammetry to $\mathrm{vrml} / \mathrm{x} 3 \mathrm{~d}$ model. In: " $3 D$ Virtual Reconstruction and Visualization of Complex Architectures", Trento, Italy 